\title{
EL FLUJO TURÍSTICO HACIA LAS TIERRAS DE LA MANCOMUNIDAD DEL MATARRAÑA (TERUEL, ESPAÑA). CARACTERÍSTICAS DE LA CLIENTELA Y DEMANDA POTENCIAL
}

\author{
Javier CALLIZO SONEIRO ${ }^{1}$ \\ Departamento de Geografía y Ordenación del Territorio \\ Universidad de Zaragoza
}

\begin{abstract}
Resumen: El Matarraña es un espacio turístico en plena expansión, cuya clientela procede tanto de la nueva y creciente demanda de turismo rural y ecoturismo, cuanto de la existencia de un intenso flujo de antiguos emigrantes convertidos hoy en residentes recreativos con carácter temporal.

El objetivo fundamental de este trabajo es aportar un intento de explicación científica de la demanda turística rural a partir del análisis de regresión de sus variables independientes: población, distancia y renta de las áreas emisoras. El análisis matemático permitió además determinar el valor de los parámetros de la ecuación y ofrecer un mapa de la demanda potencial.
\end{abstract}

Palabras clave: Matarraña, ecoturismo, turismo rural, demanda potencial, modelos teóricos.

\begin{abstract}
The county of Matarraña (province of Teruel, Spain) is a tourist space in full expansion whose customers proceed so much of the new and growing demand of rural and ecological tourism, as from the presence of an intensive flow of former emigrants who are becoming temporary recreational residents today.

The basic purpose of this paper is providing a tentative scientific explanation of rural tourist demand through regression analysis of the independent variables: population, distance and income level in the areas originating that flow. Furthermore, the matematical analysis has allowed to determine the value of the equation parameters and to offer a map of the potential demand.
\end{abstract}

Key words: Matarraña, ecological tourism, rural tourism, potential demand patterns.

${ }^{1}$ Con la colaboración del Ldo. Antonio LACOSTA ARAGUES, becario de investigación y becario ERASMUS. 
El estudio de la Mancomunidad del Matarraña como foco turístico receptor nos ha permitido verificar algunas de las hipótesis de que se sirve habitualmente 1 a geografía del turismo, pero nos ha deparado también no pocas sorpresas.

El presente artículo, que forma parte de un estudio más amplio sobre el proceso de formación de ese espacio turístico, pretende arrojar un poco de luz sobre los problemas de predicción de la demanda en el caso de los focos turísticos menores, donde la variable dependiente es explicada -entre otras- por una variable independiente - la distancia- deformada tanto por el papel de la clientela cautiva con evidentes vinculaciones afectivas -los emigrantes, convertidos ahora en residentes secundarios-, cuanto por el fuerte tropismo rural de la clientela extranjera.

Los datos proceden de una encuesta ${ }^{2}$ que se llevó a cabo entre el verano de 1993 y la primavera de 1994. El propósito fundamental era conocer las características de la clientela turística de acuerdo con sus rasgos económicos, sociales y espaciales; por otra parte, las respuestas de los visitantes nos revelaron además la estructura de este espacio turístico incipiente, información que, en geografía del turismo, sólo es posible reunir mediante un trabajo de campo de esa naturaleza.

Hasta ahí el análisis descriptivo del flujo. En la segunda parte del artículo hemos tratado de realizar un intento de explicación científica mediante la construcción de ecuaciones de regresión logarítmica múltiple a partir de la distancia al foco emisor y la población y la renta per cápita de éste como variables independientes.

La modelización tenía una doble finalidad: establecer un método de predicción de la demanda esperada que permitiese a los agentes económicos y sociales saber hacia dónde debían canalizar selectivamente sus esfuerzos de comercialización turística, pero también contribuir al conocimiento científico general de los parámetros con que las variables están afectadas. En definitiva: determinar el peso que en la ecuación tienen las diferentes variables estadísticas.

\footnotetext{
2 Universo muestral: 630 personas entrevistadas en diversos núcleos de la Mancomunidad del Matarraña, de forma aleatoria. De acuerdo con la Organización Mundial del Turismo (OMT), en su definición del viaje turístico, la estancia de al menos veinticuatro horas fuera del domicilio habitual -lo que supone al menos una pernoctación fuera de éste; de lo contrario, el viaje tendría carácter de mera excursión- ha sido requisito para poder responder a la encuesta.

Los núcleos en que se han efectuado las entrevistas, el número de éstas, así como las fechas de realización, son las siguientes: Valderrobres: 156 encuestas, 3 y 4 /VIll/1993; Beceite: 150 encuestas, 5 y 6/VIII/1993; Cretas: 60 encuestas, 7/VIII/1993; Calaceite: 150 encuestas, 9 y 11/VIII/1993; Lledó: 6 encuestas, 9/VIII/1993; Arens de Lledó: 18 encuestas, 9/VIII/1993; Peñarroya de Tastavins: 30 encuestas, 1/IV/1994; Monroyo: 15 encuestas, 1/IV/1994; Ráfales: 9 encuestas, 1/IV/1994; La Portellada: 18 encuestas, 2/IV/1994; La Fresneda: 18 encuestas, 2/IV/1994.
} 


\section{ESTRUCTURA SOCIO-ESPACIAL DE LA CLIENTELA}

A la hora de analizar el flujo turístico que acude al Matarraña, nos pareció importante conocer el grado de fidelidad de la clientela (Fig. 1). Pues bien: el 39 por 100 de la población visitó la comarca antes de 1970 (normalmente por ser su lugar de nacimiento), dato que encaja casi milimétricamente con la cifra que aporta la suma de los datos de los dos últimos intervalos de edad (37,2 por 100). Así mismo, el 26,3 por 100 declaraba haber visitado la comarca por primera vez en 1991 o en años posteriores (el 13,9 por 100 lo hacía por primera vez en el momento de ser encuestado), lo que está en estrecha relación con el exceso de personas cuyas edades se enmarcan en el intervalo 3044 años (41,4 por 100), y que constituyen el grueso de la población turística.

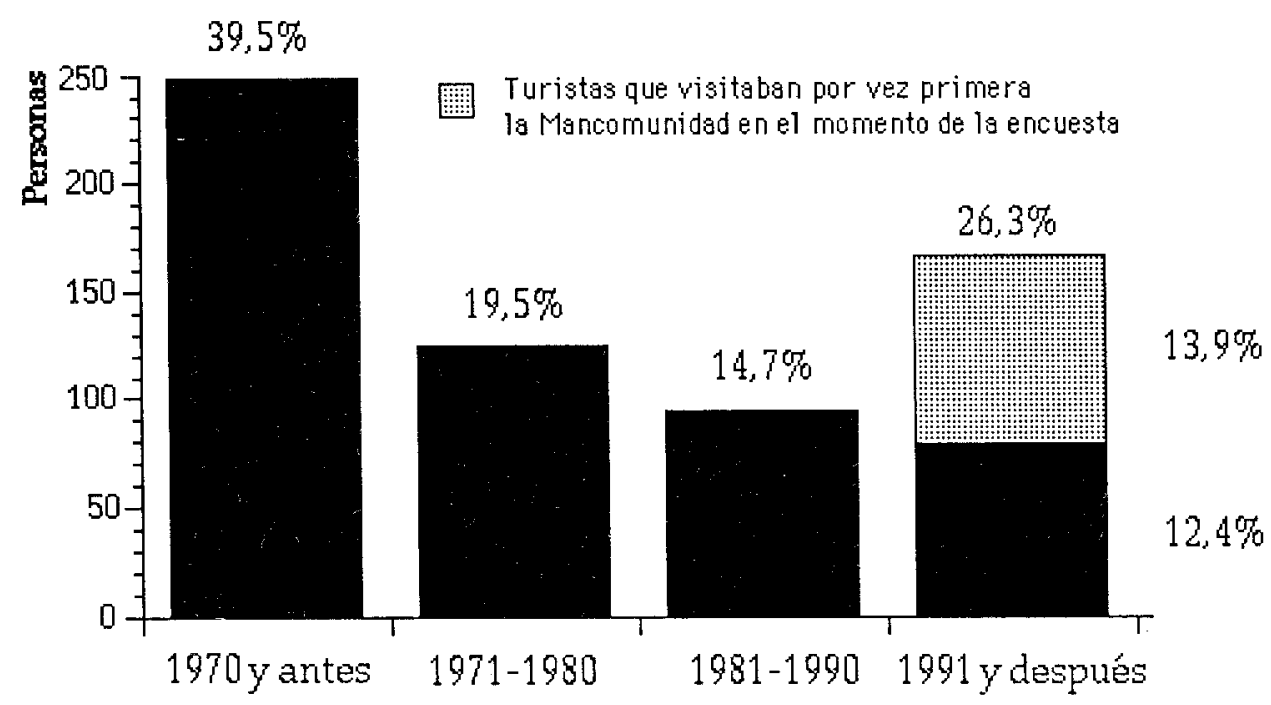

Figura 1.- Clientela turística según el momento de su primer viaje a la Mancomunidad.

Fuente: Encuesta sobre la demanda turística de la Mancomunidad del Matarraña, 19931994. Elaboración propia.

\section{Características sociales y económicas}

La clientela turística de la Mancomunidad aparece dividida en dos grupos:

a) la mayor parte de la población turística se encuentra ligada por lazos afectivos a la comarca: emigrantes nativos, más o menos recientes, y descendientes de segunda y hasta de tercera generación; 
b) el resto, lo que podríamos definir como la demanda turística no demográficamente vinculada a la Mancomunidad, se reparte entre: el grupo de los visitantes extranjeros; el de edad comprendida entre 30-44 años (viaje en familia y que es el grupo mayoritario) y el de los jóvenes de entre 15-29 años (en su mayoría, viaje en grupo de amigos o parejas). La atracción turística tiene aquí unas bases distintas de las afectivas, lo que significa también que estamos ante un segmento de la demanda cuya fidelidad depende sobre todo de la calidad del producto turístico ofrecido.

Es igualmente importante considerar que el 82,8 por 100 de los encuestados viaja en familia, advirtiéndose la presencia dominante de tres tipos de familias: a) las constituidas por parejas sin hijos, generalmente jóvenes $(19,5$ por 100$)$; b) las que cuentan con entre tres y cinco miembros (con 53,9 por 100, son el grupo más importante a la vez que definen el tipo medio de familia española actual, nuclear y constituida por el matrimonio y dos o tres hijos a lo sumo); $y$, por último, c) las que exceden de cinco miembros (4,3 por 100$)$.

Están por último los que viajan en grupo (12,9 por 100). Dentro de éstos destacan los que viajan en pareja, integrada ésta generalmente, aunque no de forma exclusiva, por personas de ambos sexos que no han establecido entre sí ningún vínculo de unión legal. Este grupo engloba al 7,1 por 100 de los encuestados, superando con mucho al resto de personas que viajan en grupos mayores (5,8 por 100). Las personas que viajan en grupo son en su mayoría jóvenes comprendidos dentro del grupo de edad de los 1.5-29 años o, en menor medida, parejas maduras y matrimonios con hijos encuadrados dentro del grupo de edad 30-44 años. No obstante, no son excepcionales parejas de personas de cierta edad.

En relación con las características socio-profesionales de la clientela (Fig. 2), el primer grupo está constituido por funcionarios, que representan el 18,6 por 100 de 1 a demanda turística. La mayoría de los municipios oscila más o menos en torno a este valor (el caso de Beceite resulta paradigmático, puesto que refleja de forma casi mimética los valores medios de la Mancomunidad para todos los grupos considerados, en este caso el 18 por 100), destacando el caso de Valderrobres, donde el grupo considerado alcanza el 27 por 100 de los turistas encuestados; Calaceite (12 por 100) y Cretas (15 por 100) presentan, por su parte, valores muy alejados de la media.

El segundo grupo socioprofesional es el integrado por los asalariados en sentido amplio. La media de la población turística en la zona es del 30 por 100, valor que presenta así mismo Beceite; destaca de entre todos los municipios Calaceite, con un 36 por 100 . 


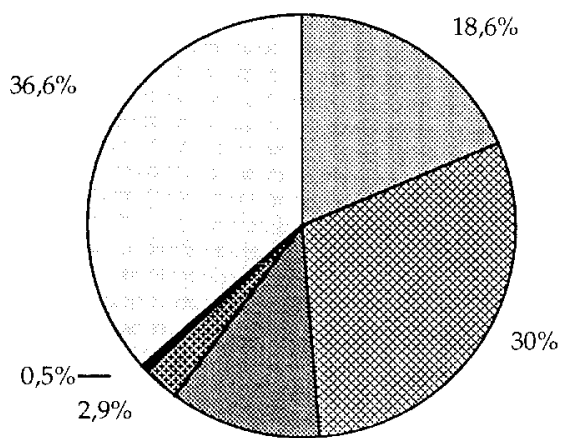

$11,4 \%$

MANCOMUNIDAD

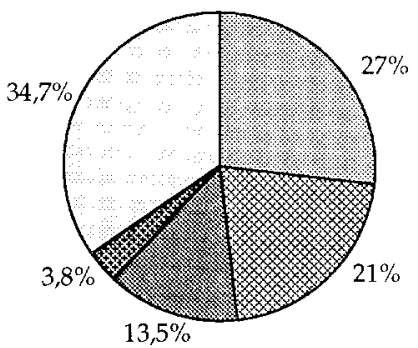

VALDERROBRES

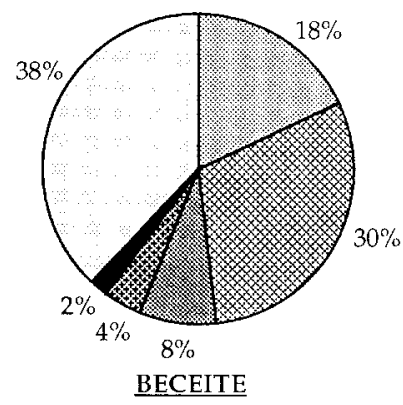

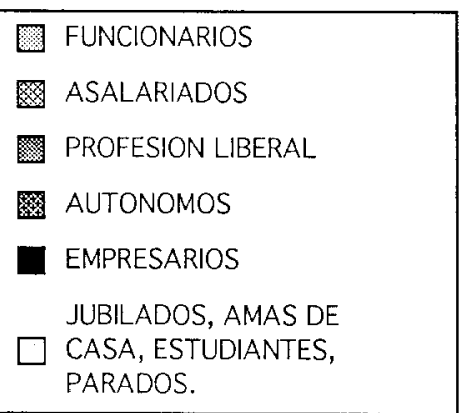

PARADOS
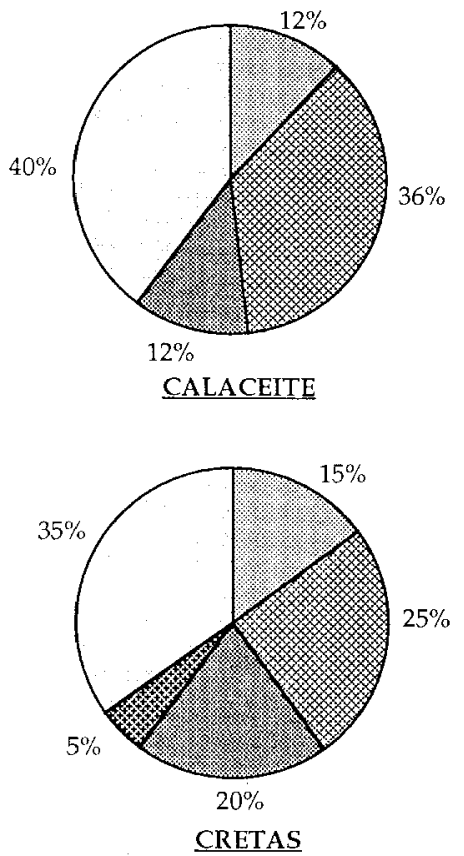

Figura 2.- Estructura socioprofesional de la clientela turística de la Mancomunidad del Matarraña.

Fuente: Encuesta sobre la demanda turística de la Mancomunidad del Matarraña, 19931994. Elaboración propia 
Las profesiones liberales representan un 11,4 por 100 del total. Dentro de este grupo, donde se encuadran las personas con mayor cualificación profesional (ingenieros, economistas, dentistas, fotógrafos, arquitectos, etc.), aparece Cretas con nombre propio, pues el grupo supone el 20 por 100 de su población turística, casi el doble que la media de la Mancomunidad; la clave parece estar en el peso que tiene aquí la emigración cualificada. Beceite, con un 8 por 100, se encuentra muy por debajo de la media; por paradójico que pueda parecer, el enclave más atractivo y turístico de la zona cuenta con una clientela menos cualificada (la explicación tiene mucho que ver con el peso de la población inactiva y con el tipo de frecuentación, según veremos luego).

Los trabajadores autónomos integran un grupo minoritario $(2,9$ por 100 de 1 a demanda turística); apenas existen en Calaceite y presentan valores un poco más elevados que la media en el resto de los municipios. En todo caso, siempre con escasa relevancia.

El grupo de los empresarios supone por último una representación meramente testimonial, en torno al 0,5 por 100 , siendo Beceite el único lugar en que se hacen presentes.

Por lo que a los inactivos se refiere, los jubilados (6,7 por 100) son un grupo menor en relación con las amas de casa y los estudiantes, que presentan valores muy similares, todos en torno al porcentaje medio de la Mancomunidad (13,3 por 100 las amas de casa y 12,4 por 100 los estudiantes). Los parados sólo suponen el 4,2 por 100 de la demanda. Los municipios analizados muestran, con leves variaciones, estas mismas proporciones.

La mayor discordancia respecto a dichos resultados aparece en Beceite. En este municipio las amas de casa y los estudiantes alcanzan los valores porcentuales más elevados, a la vez que se registra la cifra más baja de jubilados (2 por 100) y la más alta de parados (8 por 100). La explicación parece estar en el tipo de tropismo de este bello entorno natural que es El Parrizal: predominio de clientela no vinculada afectivamente a la comarca, joven, con escasos recursos económicos y con preferencia por el acampamento como forma de alojamiento (ahí se localiza precisamente la única zona de acampada libre con cierta capacidad).

Finalmente, es preciso subrayar los rasgos más característicos de la clientela extranjera. Se trata de personas maduras (el 61,5 por 100 está comprendido entre 30 y 44 años), que realizan el viaje en pareja ( 46,1 por 100$)$ o en familia $(38,5$ por 100$)$. Su nivel socioeconómico es elevado como se verá más adelante al tratar los niveles de gasto en la frecuentación, aunque ello puede desprenderse de su cualificación socio- 
profesional: un 53,8 por 100 se encuadra dentro de la función pública (30,7 por 100 dentro del mundo de la educación), un 15,4 por 100 engloba a jubilados y estudiantes y el resto, un 30,8 por 100 , se dedica a trabajos en el sector privado (agentes comerciales, empleados, etc., destacando un pequeño grupo de profesionales liberales dedicados a 1 campo de la imagen: fotografía y video)

\section{Origen espacial de la clientela}

Como se aprecia en el mapa de la Figura 3, la clientela turística que satisface sus necesidades de ocio en la Mancomunidad del Matarraña proviene en un 93,8 por 100 del territorio nacional, en su mayor parte de las provincias del Valle del Ebro y de 1 a mitad septentrional del levante español, y en un 6,2 por 100, de los paises europeos, cifra modesta que, sin embargo, no debe llevarnos a subestimar la importancia creciente de este turismo extranjero en las áreas de interior de algunos paises europeos, ${ }^{3}$ entre ellos el nuestro.

A escala nacional, la provincia de Barcelona concentra el 61,9 por 100 del total de turistas encuestados, hecho éste que se explica por la fuerte atracción demográfica ejercida desde la postguerra pero especialmente durante la década del desarrollismo (años 60 y 70) sobre la población emigrante del Matarraña. De ahí que el turismo proveniente de esta provincia presente una fuerte componente de personas con vínculos familiares o afectivos en la Mancomunidad (emigrantes o hijos de éstos en segunda y hasta tercera generación, que conservan todavía familiares o cuando menos su residencia paterna convertida en secundaria). Como muestra, un botón: en Valderrobres el 86 por 100 de los visitantes radicados en la provincia de Barcelona se alojaba en casa de familiares o residencia secundaria; en Calaceite lo hacía el 68,6 por 100 (si bien un 14,3 por 100 más lo hacía en casa de amigos, lo que es reflejo fiel de que se ha producido ya un proceso de difusión); en Peñarroya, el 50 por 100; Beceite presenta la principal novedad ya que tan solo el 39,4 por 100 de los barceloneses se alojaba en casas de familiares o residencias secundarias, mientras que um 30,3 por 100 lo hacía en casas alquiladas.

Un segundo grupo de provincias con presencia turística importante en la zona lo integran Zaragoza ( 9 por 100) y Tarragona (7,1 por 100). En estos casos, tan importante resulta el componente de turistas con lazos afectivos como el de los demográficamente no vinculados y que se acercan animados por la proximidad de esta comarca a sus lugares de residencia habitual.

\footnotetext{
3 Véanse los trabajos de BULLER y HOGGART (1994) sobre el flujo turístico británico en el espacio rural francés.
} 

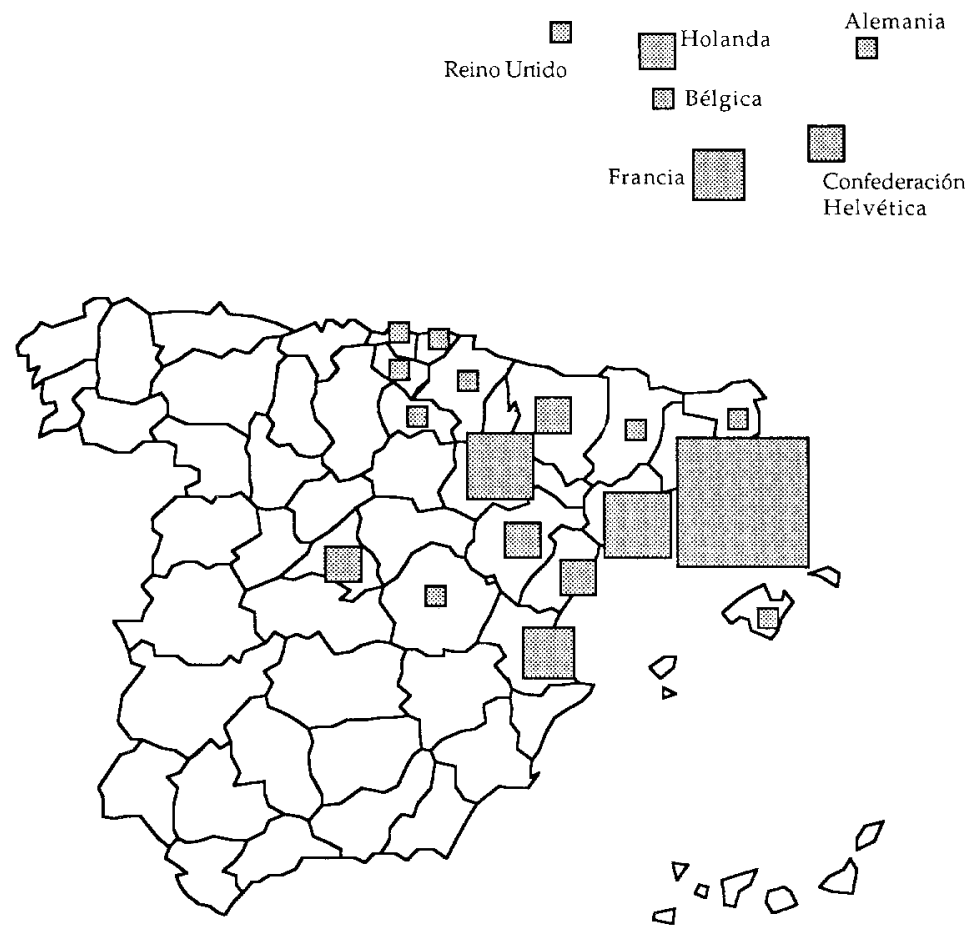

Número de turistas

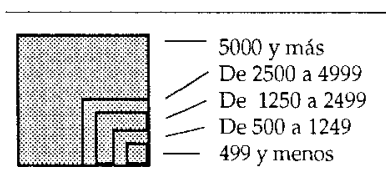

Figura 3.- Origen de la clientela turística del Matarraña, 1994.

Fuente: Encuesta sobre la demanda turística de la Mancomunidad del Matarraña. Elaboración propia.

El tercer conjunto de provincias por el número de visitantes registrados en 1 a encuesta reúne a las de Valencia (3,8 por 100), Castellón (2,8 por 100), Teruel (2,4 por $100)$ y Madrid (1,4 por 100). En los dos primeros casos, la proximidad a la zona y una cierta accesibilidad explican en buena medida el importante flujo turístico; explicación a la que conviene añadir la alterotropía que para este tipo de clientela supone pasar de un medio urbano costero a otro montañoso interior. De Madrid, sin 
embargo, el turismo registrado es fundamentalmente de tipo ocasional (66 por 100), hallándose en una buena proporción en ruta por las serranías de Cuenca y Teruel (la mayoría de los turistas fueron entrevistados en Beceite y están en sintonía con las características tipológicas de la frecuentación de El Parrizal, anteriormente señaladas). El flujo turolense, finalmente, se reparte así: el 60 por 100 proviene de Alcañiz o pueblos cercanos y un 40 por 100 de Teruel capital, teniendo algún tipo de relación con la Mancomunidad (familiar, profesional, etc.); las malas comunicaciones con el resto de la provincia y la escasa alterotropía que para el resto de los turolenses tiene este espacio natural respecto de sus lugares habituales de residencia, 1o convierten en un objetivo poco atractivo.

Por último, están aquellas provincias con una presencia tal vez anecdótica (menos del 1 por 100). El abanico es amplio: Lérida, Gerona, Vizcaya, Guipúzcoa, Alava, Navarra, La Rioja, Cuenca, Baleares y Huesca (esta última destaca sobre el resto de las que se agrupan en este segmento de la demanda con un 0,9 por 100 ).

Antes de proceder a formular una hipótesis explicativa, hemos querido agotar la metodología analítica, ponderando la variable dependiente a partir de la variable población del foco emisor, de acuerdo con el sencillo método del ratio espacial en tantos por cien mil utilizado por CHADEFAUD (1971), y que se formula así:

$$
\mathrm{R}=\frac{\mathrm{N}_{\mathrm{e}}}{\mathrm{N}_{\mathrm{t}}} 10^{5}
$$

donde: $R$ = ratio del origen espacial

$N_{\mathcal{C}}=$ número de turistas de una provincia $N$

$N_{r}=$ número de habitantes de dicha provincia $N$

Pues bien, Teruel y Barcelona representan, junto a Tarragona, las provincias más intensamente atraídas por el Matarraña (Fig 4); ahora bien, por su escaso volumen demográfico, Teruel, con un 10,59 por 100.000, alcanza sin embargo una tasa de origen relativo mucho mayor que la de Barcelona (8,31 por 100.000). Algo similar se advierte también en los casos de Tarragona y Zaragoza: ésta aporta un 2 por 100 más de turistas que aquélla, pero al poseer una población mucho mayor su importancia relativa desciende, de ahí que en la cartografía aparezca en una situación intermedia entre los focos más importantes y los que presentan valores próximos a la media: Castellón, Cuenca y Huesca. El resto de las provincias se engloba dentro del arco de valores comprendidos entre $-0,5 \mathrm{~s}(1,21$ por 100.000$)$ y $-1,5 \mathrm{~s}$ (valor que está por debajo de 0 ): en 
todas ellas el número de visitantes registrado, dada su componente de ocasionalidad, es lo suficientemente bajo como para no suponer un grupo significativo dentro de su provincia.

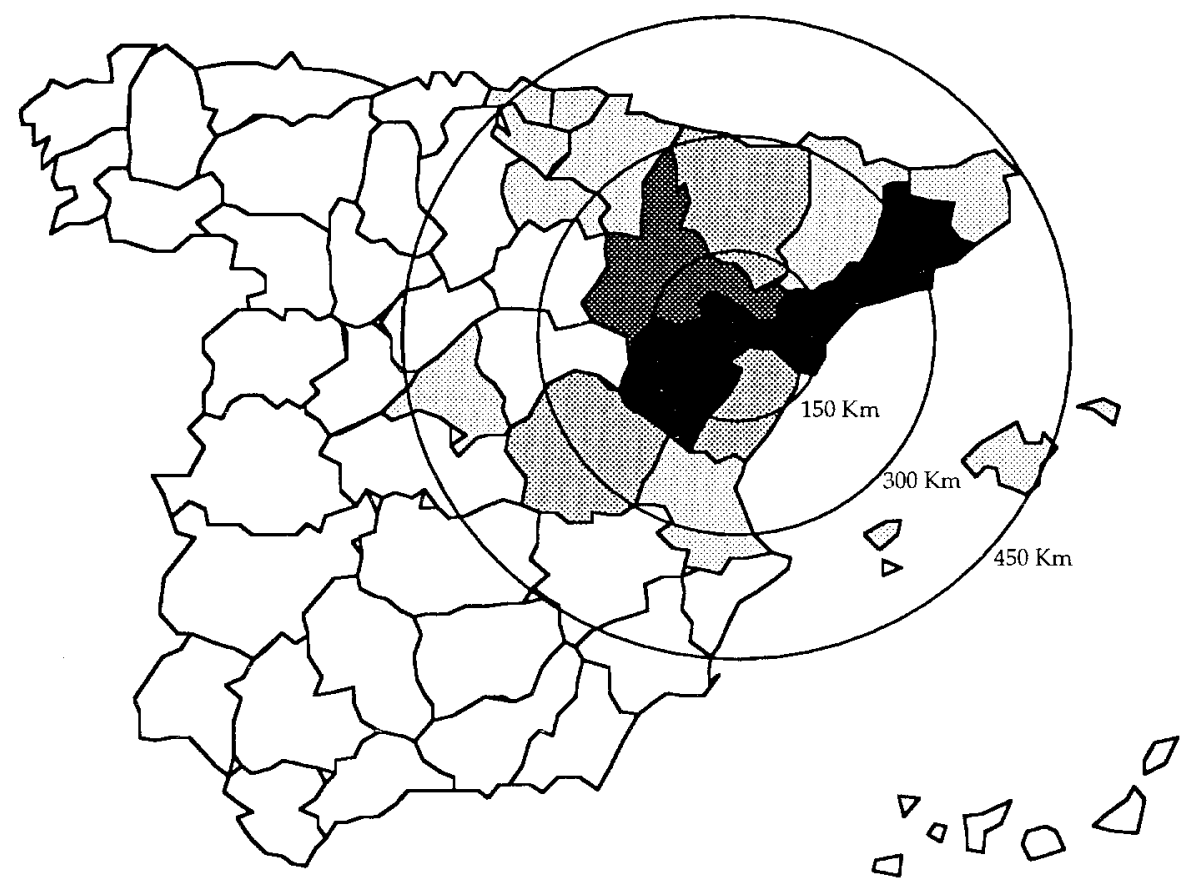

Ratio de frecuentación (Ri) por 100.000 habitantes (Ratio de Chadefaud)

Discretización a partir de la desviación típica

$\overline{\mathrm{X}}=2.86 ; \sigma=3.30$

$\mathrm{Ri}=(\mathrm{Ti} / \mathrm{Pi}) 100.000$

$\mathrm{Ti}=$ Turistas procedentes de la provincia $\mathrm{i}$

$\mathrm{Pi}=$ Población de la provincia $\mathrm{i}$

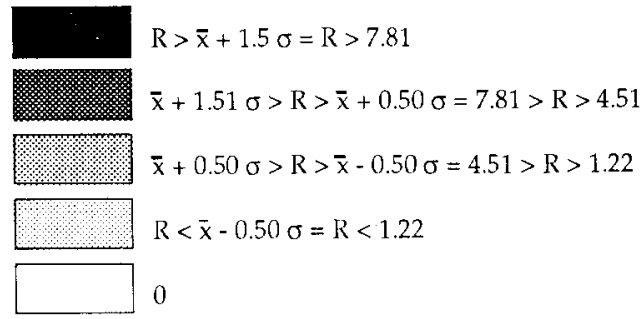

Figura 4.- El área de influencia turística del Matarraña, 1994.

Fuente: Encuesta a la clientela turística de la Mancomunidad del Matarraña. Elaboración propia. 
El mapa revela, pues, la existencia de un área de influencia turística cuyo gradiente se deforma en el Matarraña de acuerdo con la interacción de tres variables:

a) el grado de vinculación demográfica de la clientela con la comarca;

b) la proximidad: el aumento de la variable "distancia" se traduce en un inmediato incremento de la desinformación sobre la Mancomunidad, de suerte que los viajeros de provincias situadas a más de $300 \mathrm{Km}$ no dicen acudir al Matarraña sino a la provincia de Teruel); y

c) la accesibilidad.

\section{EVOLUCION Y ORIGEN DEL FLUJO TURISTICO POTENCIAL}

Hasta aquí el análisis descriptivo del flujo turístico recibido por el Matarraña. En los párrafos que siguen trataremos, además, de "explicar" para poder finalmente predecir. Conocidos, pues, los parámetros de la ecuación, ¿cómo evolucionará el flujo futuro?, ¿cuál será su distribución espacial hipotética?. Vayamos con la primera cuestión.

\section{Los horizontes evolutivos del flujo potencial}

Como es metodológicamente habitual en este tipo de explicaciones matemáticas, hemos partido de la variable turistas observados en los años censales pasados, sometida a un análisis de regresión de dos tipos -simple y polinomial-, que representan otros tantos escenarios de demanda potencial, según puede apreciarse en las tablas 1 y 2 y en la Figura 5.

Los valores de frecuentación del último año estudiado nos dan un volumen en torno a los 58.465 turistas, cifra que apenas se aparta de las estimaciones ofrecidas por la Asociación de Turismo Rural de la Mancomunidad. Las estimaciones de los años anteriores (hasta 1970) han sido obtenidas de forma indirecta a partir de una encuesta a determinados comerciantes cuya actividad está altamente vinculada con el turismo (el excursionismo incluido).

Pues bien, en los dos tipos de regresión se aprecia una previsión más positiva a partir de 1997 que antes de esa fecha; lo que no es incomprensible si pensamos que 1 a predicción estadística está acusando los efectos de la notable caída de la frecuentación en el año 1992, como consecuencia de la canalización de una parte muy caudalosa del 
flujo potencial hacia los dos megaevents de moda, a la sazón: los Juegos Olímpicos de Barcelona y la Exposición Universal de Sevilla.

Tabla 1.- Demanda turística de la Mancomunidad del Matarraña.

Frecuentación reciente y recepción potencial.

Fuente: Asociación de Turismo Rural de la Mancomunidad del Matarraña y Encuesta a la Clientela. Elaboración propia.

\begin{tabular}{|c|c|c|c|}
\hline Año & $\begin{array}{c}\text { Turistas } \\
\text { observados (To) }\end{array}$ & $\begin{array}{c}N^{\circ} \text { Turistas } \\
\text { esperados (Te) } \\
\text { (Regres. polinom) }\end{array}$ & $\begin{array}{c}N^{\circ} \text { Turistas } \\
\text { esperados (Te) } \\
\text { (Regres. simple) }\end{array}$ \\
\hline 1970 & 20463 & 21482 & 13680 \\
\hline 1980 & 17540 & 16646 & 24628 \\
\hline 1985 & 28648 & 22243 & 30102 \\
\hline 1990 & 33325 & 33238 & 35576 \\
\hline 1991 & 30402 & 36088 & 36671 \\
\hline 1992 & 23386 & 39156 & 37766 \\
\hline 1993 & 45018 & 42442 & 38861 \\
\hline 1994 & 58465 & 45947 & 39956 \\
\hline 1995 & & 49670 & 41050 \\
\hline 1996 & & 53613 & 42145 \\
\hline 1997 & & 57775 & 43240 \\
\hline 1998 & & 62157 & 44335 \\
\hline 1999 & & 66759 & 45430 \\
\hline 2000 & & 71581 & 46525 \\
\hline 2005 & & 99011 & 51999 \\
\hline
\end{tabular}

Con un coeficiente de correlación de 43 por 100, propio de las fuertes fluctuaciones de la serie, el escenario más pesimista nos da unas perspectivas de crecimiento moderadas, y desde luego más evidentes a medio plazo: el volumen de turistas no sería inferior a 50.000 en el año 2000, y podría superar decidida y sostenidamente la cifra actual hacia el año 2020. 
Tabla 2.- Origen del flujo turístico hacia la Mancomunidad del Matarraña en 1995. Clientela observada y demanda esperada.

Fuentes: I.N.E., Censo de Población de 1991; Banco de Bilbao: Renta Nacional de España y su distribución provincial. Elaboración propia.

\begin{tabular}{|c|c|c|c|c|c|c|}
\hline Provincia & $\begin{array}{l}\text { Renta 'per } \\
\text { cápita' }\end{array}$ & Población & Distancia $\mathrm{Km}$ & $\begin{array}{c}\text { Turistas } \\
\text { observados } \\
\text { (To) }\end{array}$ & $\begin{array}{c}\text { Turistas } \\
\text { esperados } \\
\text { (Te) }\end{array}$ & To-Te \\
\hline Alava & 14388 & 272447 & 350 & 302 & 138 & 164 \\
\hline Albacete & 8014 & 342677 & 327 & 0 & 256 & -256 \\
\hline Alicante & 10792 & 1292564 & 380 & 0 & 650 & -650 \\
\hline Almería & 7999 & 455496 & 674 & 0 & 90 & 90 \\
\hline Asturias & 9569 & 1093937 & 734 & 0 & 172 & -172 \\
\hline Avila & 7868 & 174378 & 536 & 0 & 52 & -52 \\
\hline Badajoz & 6205 & 650388 & 781 & 0 & 110 & -110 \\
\hline Baleares & 14838 & 709137 & 280 & 277 & 541 & -264 \\
\hline Barcelona & 14204 & 4654410 & 200 & 38678 & 6858 & 31820 \\
\hline Burgos & 11351 & 352771 & 430 & 0 & 136 & -136 \\
\hline Cáceres & 8311 & 411465 & 677 & 0 & 79 & -79 \\
\hline Cádiz & 7215 & 1078403 & 1043 & 0 & 101 & -101 \\
\hline Cantabria & 10062 & 527324 & 527 & 0 & 148 & -148 \\
\hline Castellón & 11815 & 446743 & 150 & 1791 & 1186 & 605 \\
\hline Ciudad Real & 8506 & 475434 & 569 & 0 & 125 & -125 \\
\hline Córdoba & 6700 & 754452 & 780 & 0 & 124 & -124 \\
\hline Coruña, La & 9534 & 1096966 & 1074 & 0 & 86 & -86 \\
\hline Cuenca & 7343 & 205198 & 250 & 302 & 260 & 42 \\
\hline Gerona & 15381 & 509627 & 300 & 290 & 336 & -46 \\
\hline Granada & 6518 & 790516 & 814 & $\begin{array}{r}0 \\
\end{array}$ & 121 & -121 \\
\hline Guadalajara & 11149 & 145593 & 320 & 0 & 97 & -97 \\
\hline Guipuzcoa & 11980 & 676488 & 370 & 304 & 339 & -35 \\
\hline Huelva & 7607 & 443476 & 1012 & 0 & 42 & -42 \\
\hline Huesca & 11455 & 207810 & 200 & 567 & 326 & 241 \\
\hline Jaén & 6804 & 637633 & 715 & 0 & 122 & -122 \\
\hline La Rioja & 12065 & 263434 & 300 & 292 & 192 & 100 \\
\hline León & 8907 & 525896 & 631 & 0 & 112 & -112 \\
\hline Lérida & 12259 & 353455 & 90 & 297 & 2362 & -2065 \\
\hline Lugo & 7746 & 384364 & 865 & 0 & 49 & -49 \\
\hline Madrid & 14203 & 4947566 & 380 & 891 & 2234 & -1343 \\
\hline Málaga & 8825 & 1160844 & 800 & 0 & 162 & -162 \\
\hline Murcia & 8967 & 1045604 & 455 & 0 & 409 & -409 \\
\hline Navarra & 12853 & 519277 & 280 & 296 & 421 & -125 \\
\hline Orense & 8204 & 353491 & 861 & 0 & 44 & -44 \\
\hline Palencia & 10623 & 185478 & 516 & 0 & 52 & -52 \\
\hline Palmas, Las & 10427 & 767969 & 2500 & 0 & 12 & -12 \\
\hline Pontevedra & 8994 & 896849 & 963 & 0 & 88 & -88 \\
\hline S.C. Tenerife & 10427 & 725814 & 2500 & 0 & 11 & -11 \\
\hline Salamanca & 8745 & 357801 & 565 & 0 & 94 & -94 \\
\hline Segovia & 9119 & 147188 & 469 & 0 & 53 & -53 \\
\hline Sevilla & 8161 & 1619701 & 918 & 0 & 182 & -182 \\
\hline Soria & 8962 & 94537 & 311 & 0 & 73 & -73 \\
\hline \begin{tabular}{|l|} 
Tarragona \\
\end{tabular} & 13974 & 542004 & 90 & 4472 & 3433 & 1039 \\
\hline Teruel & 10786 & 143680 & 80 & 1522 & 1251 & 271 \\
\hline Toledo & 9029 & 459542 & 450 & 0 & 193 & -193 \\
\hline Valencia & 11799 & 2117927 & 210 & 2372 & 3072 & -700 \\
\hline Valladolid & 11894 & 494207 & 450 & 0 & 173 & -173 \\
\hline Vizcaya & 11303 & 1155107 & 400 & $2 \pi$ & 517 & -240 \\
\hline Zamora & 7719 & 213688 & 546 & 0 & 63 & -63 \\
\hline Zaragoza & 12182 & 837328 & 130 & 5535 & 2872 & 2663 \\
\hline
\end{tabular}




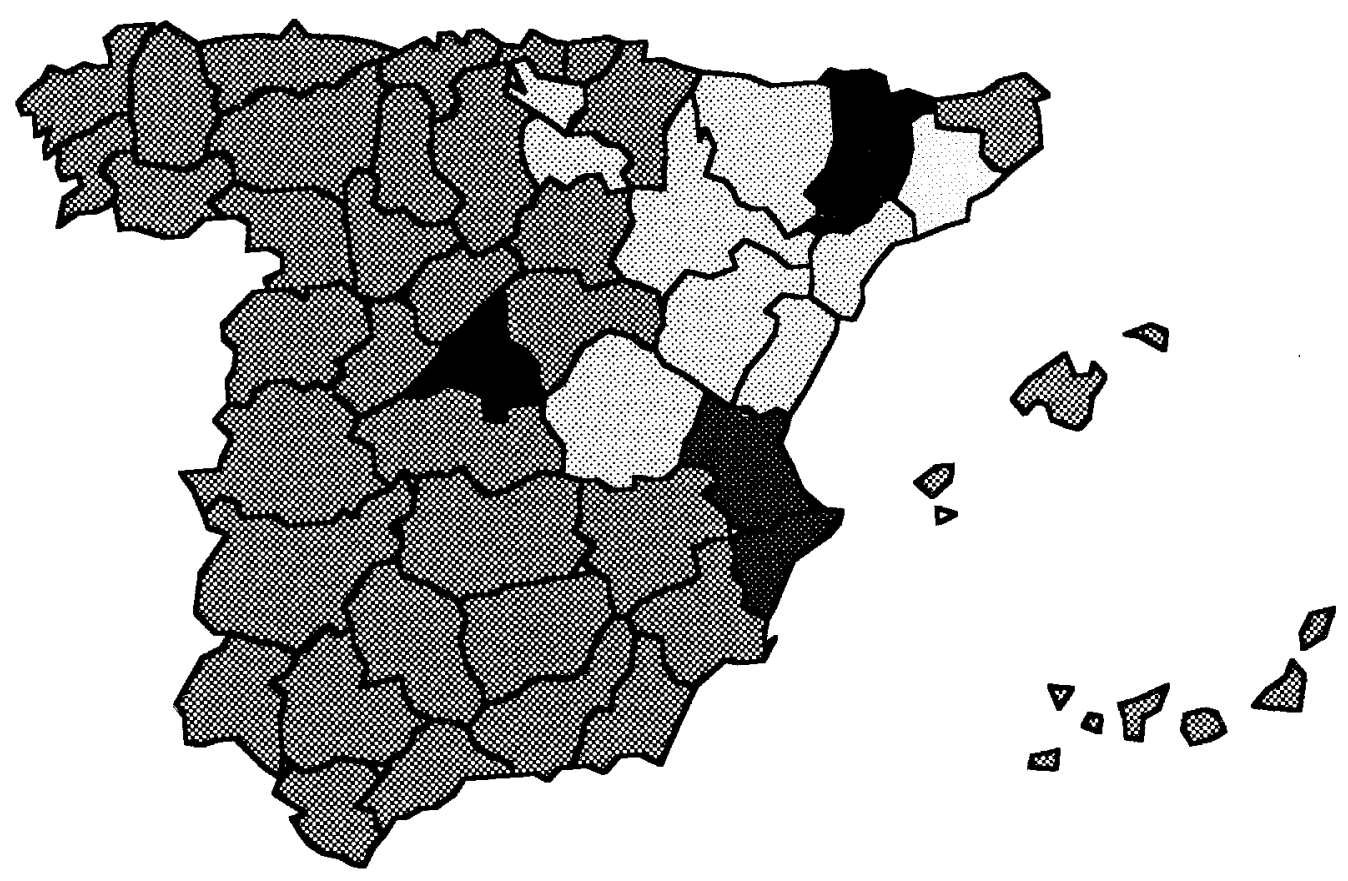

DEMANDA TURISTICA POTENCIAL

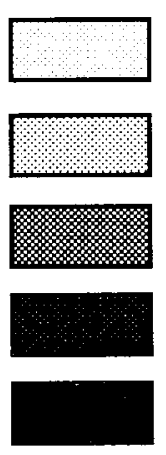

5000 y más

a a

De 0 a 4999

De 0 a -499

De -500 a -999

-1000 y menos
$\mathrm{Dp}=\mathrm{To}-\mathrm{Te}$

$\mathrm{Dp}=$ Demanda potencial

To $=$ Turistas observados

$\mathrm{Te}=$ Turistas esperados $=\mathrm{y}$

$\mathrm{y}=1514\left(\mathrm{P}^{\wedge} 1.01\right)\left(\mathrm{R}^{\wedge}-0.442\right)\left(\mathrm{D}^{\wedge}-1.844\right)$

$\mathrm{R}=$ Renta 'per cápita' prov. emisora

$\mathrm{P}=$ Población prov. emisora

$\mathrm{D}=$ Distancia Matarraña-Prov. emisora

Figura 5.- La demanda turística potencial de la Mancomunidad del Matarraña, 1995. Fuentes: I.N.E., Censo de Población de 1991; BANCO DE BILBAO, Renta Nacional de España y su distribución provincial, y Encuesta para el estudio del potencial turístico de la Mancomunidad del Matarraña. Elaboración propia. 
El escenario más optimista -por otra parte es el que presenta una correlación estadística más alta, del orden de 94,9 por 100 - permite superar la cifra actual de visitantes a partir del año 1997, de modo que en 1998 la clientela estimada asciende a 62.157, y para el año 2005 asistiríamos casi a la duplicación de la última de las cifras de frecuentación observadas (99.011 turistas). Es claro que las previsiones para este año y para el siguiente son optimistas: aunque el ajuste estadístico prevea lo contrario, 1 a inflexión del año 92 -como se ha escrito con anterioridad-, está lastrando a la baja las previsiones para los años inmediatamente posteriores al momento de la crisis.

Este último escenario tiene, pues, visos de gran verosimilitud (así lo indica su altísimo índice de correlación). Ahora bien, sobre una tendencia al alza que nos permite ser más optimistas que nunca, no es bueno olvidar que la historia del turismo está llena de crisis coyunturales -y también estructurales- no pocas veces imprevisibles. Al fin y a la postre, la distribución espacial del flujo turístico no puede ser explicada fuera de las interacciones globales del sistema turístico mundial: una mejora sustancial de la paridad de la peseta puede tener efectos muy catastróficos sobre el turismo de interior y también sobre el turismo rural, al reconducir el flujo potencial hacia mercados internacionales al tiempo que el nuestro pierde en las ventajas monetarias una parte muy destacada de la atracción en que se apoya. El turismo rural, tan pujante en los tres últimos años, puede quedar entonces reducido a 1 a condición de flujo coyuntural de reserva, típico de momentos de depresión económica; mal haríamos en no tomar en serio al mercado turístico mundial o en pensar que el nuestro, por tratarse de un foco menor, está al socaire de los vaivenes de aquél.

\section{El valor de los parámetros de la ecuación. Una reflexión teórica}

Tras la hipótesis de frecuentación futura, nos ocuparemos seguidamente del peso de las variables que puedan explicar el origen espacial de la clientela. En este caso hemos procedido a construir una ecuación de regresión logarítmica como método para determinar luego la población turística potencialmente esperada $T_{e}$ en la Mancomunidad.

Los pasos han sido los siguientes:

a) Definición de las variables estadísticas independientes $x_{1}, x_{2}$ y $x_{3}$ transformadas logarítmicamente: población $(\log P)$ y renta per cápita $(\log R)$ del foco emisor, así como distancia $(\log D)$ desde éste a la Mancomunidad del Matarraña.

b) Regresión múltiple de la variable logarítmica dependiente -población turística, es decir, $\log y$ - a partir de las tres variables independientes referidas. 
c) Obtención de los coeficientes de regresión como base de la ecuación y cuya construcción resultó ser la siguiente:

$$
y=1514 \frac{P^{1.01}}{R^{0.44} D^{1.844}}
$$

Desde el punto de vista del análisis estadístico, es preciso añadir que esta ecuación ha sido sometida a dos tipos de test de significación: el $t$ de Student para los coeficientes de regresión logarítmica, que constituyen los parámetros de cada una de las variables, y un análisis de varianza (ANOVA) mediante la distribución $F$ de Snedecor. Los resultados son los siguientes:

a) las variables $\log P$ y $\log D$ son estadísticamente significativas y en ambos casos puede rechazarse la hipótesis nula $H_{O}$ (la probabilidad de que los resultados se hayan obtenido por azar es, respectivamente de de 0.0017 y de 0.0014);

b) el valor del parámetro $R$ no es ya tan significativo: no puede rechazarse $H_{O}$ y hay una probabilidad 0.7 de que el resultado se haya obtenido aleatoriamente; sensu contrario, lo que desde luego no puede afirmarse es que $T_{e}$ sea una función directa de $R$, como sí lo es de $P$;

c) la ecuación es en conjunto estadísticamente significativa: F está muy por encima de los valores críticos señalados para los grados de libertad $v_{1}$ y $v_{2}$ puede rechazarse $H_{0}$ y la probabilidad de que el resultado tenga un origen aleatorio es tan sólo de 0.0020 , por lo que la bondad de la ecuación está fuera de dudas.

No sin cierta sorpresa, pues, la variable dependiente (número de turistas: $T_{\mathcal{C}}=y$ ) es una función directa de la población de las provincias emisoras y simultáneamente una función inversa de la renta 'per cápita' y de la variable distancia, cuya fricción en este tipo de productos turísticos se revela determinante a partir de la isoalterotropa de los $500 \mathrm{Km}$ (a partir de esa distancia, el producto "Matarraña" deja de ser atractivo para el turismo nacional, que desaparece en beneficio de una clientela internacional, para la que el turismo rural, más allá de la citada isoalterotropa, viene ya siendo una opción interesante desde hace unos años). Estamos pues ante una explicación científica del tipo de los modelos de gravedad, tan familiares en geografía humana y desde luego en geografía del turismo, modelos del tipo:

$$
T_{e}=G P^{a} D^{-d} \text { y en este caso, } T_{e}=G P^{a} R^{-r} D^{-d}
$$


Son legión ya los autores que han aplicado en alguna de sus formas este tipo de razonamiento matemático, desde REILLY (1929) y STEWART (1947) y ZIPF (1949), pasando por VAN DOREN (1967), WOLFE (1972), MCEVOY (1974), KLAASEN (1974), BIJKERK (1975), VAN LIER (1977), SMITH (1983) y más recientemente en España ESTEBAN TALAYA (1987). La mayoría de ellos han puesto el acento en el papel de fricción de la variable distancia; sus parámetros van desde el newtoniano $\mathrm{d}=-2$ de Reilly, al $\mathrm{d}=-1$ de Stewart-Zipf, más propio de un país como Estados Unidos donde esta variable ejerce una fricción mucho menor; pocos sin embargo han ponderado otras variables socioeconómicas como hemos hecho en nuestro caso con la renta per cápita.

Nuestra aportación científica ha querido contribuir, pues, a cubrir la laguna existente en el conocimiento de las características espaciales de los flujos turísticos rurales, que, en lo que se nos alcanza, no han sido todavía objeto de este tipo de explicación. El valor de los parámetros ha revelado en nuestro caso:

a) Una fricción de la distancia $D$ próxima - pero sensiblemente inferior- a 1 a de la ecuación newtoniana (d=-2) observada por REILLY (1929), y un poco mayor que la obtenida por SMITH (1983) en un estudio sobre la clientela turística de Ontario (d=1.67), y que es expresión del diferente concepto que de la distancia tiene el consumidor norteamericano, según se acaba de referir recordando a STEWART (1947) y ZIPF (1949); en nuestro caso, d=-1.844 explica tanto la rarefacción del flujo más allá de 1 a isoalterotropa de los $500 \mathrm{Km}$, cuanto la reaparición del mismo por encima de la isoalterotropa de los $1.000 \mathrm{Km}$ (Miossec había llegado en 1976 a resultados análogos al estudiar la dilatación mundial del turismo alemán); en definitiva, como ya se $\mathrm{h}$ a escrito, a partir de la primera isolinea el turismo nacional desaparece en beneficio de la clientela extranjera, para la que el papel de la distancia es en este estadio de su evolución recreativa mucho menos relevante;

b) La población $P$ sigue siendo una de las variables más importantes para explicar la magnitud de la frecuentación turística; en este caso, y como en casi todos los fenómenos a los que se ha aplicado (área de influencia urbana, sobre todo), a resulta ser igual a 1.01 ;

c) Con todas las reservas que exige el hecho de que no pueda rechazarse $H_{0}$, este tipo de flujo turístico -el turismo rural, sobre todo-, no es una función directa de la renta per cápita $R$; muy al contrario de lo que algunos han querido ver -movidos sobre todo por un irreflexivo aunque no incomprensible desdén hacia las fórmulas de sol y playa-, y con todas esas cautelas, este tipo de frecuentación se explica más bien como una función inversa de la renta $(\mathrm{r}=-0.442)$; digamos, pues, que las expectativas derivadas de una gran concentración demográfica en el foco emisor quedarían parcialmente 
frenadas por una renta per cápita alta; o que la distancia vería incrementada su fricción en el caso de rentas altas ${ }^{4}$.

\section{La demanda esperada y su distribución espacial}

Con la ecuación de regresión múltiple obtenida se ha calculado la población turística esperada de las distintas provincias españolas; los datos se recogen en la tabla y el mapa adjuntos. Del ajuste estadístico hemos extraído las siguientes conclusiones:

1) Barcelona es, de lejos, el principal foco emisor, pero debido sobre todo al peso de la clientela con vínculos afectivos y demográficos -antiguos emigrantes hoy residentes secundarios-, su volumen aparece fuertemente sobreestimado en relación con las previsiones. Lo que significa ni más ni menos que la clientela observada está muy por encima de la potencialmente esperada; por lo que parece poco útil orientar en aquella provincia los esfuerzos futuros de comercialización (no es evidente que esa publicidad suplementaria fuera más eficaz que la difusión de boca en boca).

2) Con valores de frecuentación observada superiores a los esperados teóricamente aparecen Alava, las tres provincias aragonesas, la Rioja, Tarragona, Castellón y Cuenca. Se trata de provincias cuyo nexo fundamental es su situación aquende la isoalterotropa de los $300 \mathrm{Km}$ y en el caso de Alava además con una indudable demanda de turismo verde; en los casos de Cuenca y Tarragona, la evidente accesibilidad se ve reforzada por el eje de tropismo rural que parece dibujarse sinérgicamente entre el confín meridional de la Cordillera Ibérica y las estribaciones de la Cordillera Costero-Catalana (Serranías de Cuenca, Albarracín, Javalambre, Gúdar, Maestrazgo y Puertos de Beceite).

3) Por debajo de las previsiones, lo que quiere decir que la clientela observada es inferior a la potencialmente esperada (entre 0 y 500 turistas), aparece la mayor parte del territorio nacional.

4) La España más potencialmente cliente del Matarraña está localizada en cuatro provincias: Lérida, Madrid, Valencia y Alicante. En la primera de ellas, la de mayor flujo potencial, la explicación parece estar en la gran proximidad a nuestra comarca de estudio, proximidad que se ve neutralizada por la escasa alterotropía que para un leridano representa en general una zona rural de media montaña; algo parecido a lo que ya advertíamos en el caso del Pirineo aragonés, respecto del origen

4 Dadas dos provincias emisoras hipotéticas A y B, con población en ambos casos de 100.000 habitantes, con distancia al Matarraña en ambos casos también de $100 \mathrm{Km}$ y con renta per cápita de 5.000 y 8.000 dólares, para A y B respectivamente, la predicción obtenida por regresión nos daría una cifra de turistas esperados de 821 para $A$ y de 667 para $B$. 
de su clientela regional (CALLIZO, 1995 y 1996). Valencia y Alicante constituyen dos focos de gran potencialidad dados su peso demográfico y su relativa proximidad; la subfrecuentación sin embargo es explicable por la fuerte atracción que para la clientela valenciana tienen otros destinos turísticos análogos (la Serranía de Albarracín o el Maestrazgo castellonense sobre todo). En Madrid, cuyo potencial emisor es en términos absolutos el mayor de España (en este caso la población turística esperada supera el millar), la subfrecuentación es producto de la gran cantidad de productos turísticos similares que entran en competencia, de suerte que la información empieza a ser mucho más determinante que el resto de las variables; y parece claro que, para la clientela madrileña, el Matarraña es todavía una experiencia turística por descubrir.

\section{CONCLUSION}

De las páginas precedentes, en que hemos pasado de la descripción al intento de explicación del flujo turístico que tiene por destino las tierras de la Mancomunidad del Matarraña, cabe extraer las siguientes conclusiones:

a) Se trata de un flujo que se explica sobre todo por la proximidad hasta la isoalterotropa de los $500 \mathrm{Km}$, allende la cual cede intensidad en beneficio de la frecuentación de origen extranjero, para la que estas modalidades turísticas vienen siendo práctica habitual desde mediados de la pasada década.

b) Desde el punto de vista socio-económico, la clientela observada muestra una gran heterogeneidad: turistas de nivel cultural mayor que sus ingresos económicos comparten destino recreativo con jóvenes e inactivos, practicantes de las nuevas modalidades de aventura.

c) El grueso de la clientela -en su mayoría flamantes residentes secundarios ahora- tiene su origen en el éxodo rural de los años sesenta. Eso explica también la sobrefrecuentación turística barcelonesa, ciertamente intensa; existe por el contrario un mercado de gran potencialidad, siempre dentro de la isoalterotropa de los $500 \mathrm{Km}$ (Madrid, Lérida, Valencia y Alicante), a donde deben dirigirse los esfuerzos de publicidad selectiva en los próximos años.

d) El valor de los parámetros de la ecuación nos habla tanto de la importancia creciente de este tipo de migración temporal (la distancia ejerce aquí una fricción sensiblemente menor que en la mayoría de los desplazamientos convencionales), cuanto del peso demográfico del foco emisor; no puede decirse lo mismo de la cualificación económica, que, aunque con escasa significación estadística, se presenta más bien como 
un factor que potencia negativamente el efecto de fricción de la distancia, frenando el caudal de la demanda turística en el medio rural. Al menos por ahora.

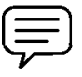

\section{BIBLIOGRAFIA}

BEAMAN, J. (1974): "Distance and the "reaction" to the distance as a function of distance", Journal of Leisure Research, 6.

BIJKERK, C. (1975): Recreation values of forest and parks. London: Phil. Trans. Royal Society.

BULLER, H. y HOGGART, K. (1994): "Vers une campagne européenne: les Britanniques en France rurale", L'espace géographique, 3, 263-273.

CALLIZO SONEIRO, J. (1995): "Las nuevas tendencias alterotrópicas del turismo en el Pirineo aragonés", La formació, la rehabilitació i les noves modalitats turístiques, III Jornades de geografia del turisme, Palma, 28-29-30 d'octubre de 1993. Palma: Servei de Publicacions de la Universitat de les Illes Balears; CODEFOC, Govern Balear; F.S.E., EUROFORM. 167-177.

CALLIZO SONEIRO, J. (1996): "Potencialidades turísticas de las áreas interiores. Conflictos y Cautelas", IV Jornadas de geografía del turismo, Toledo, 23-24-25 de marzo de 1995. Madrid: Asociación de Geógrafos Españoles (en prensa).

CHADEFAUD, M. (1971): "Méthode d'analyse d'un flux touristique au niveau local et régional", Les Cahiers du Tourisme, serie C, 17.

ESTEBAN TALAYA, A. (1987): Análisis de la demanda: aplicación a la actividad turistica de las técnicas de predicción. Madrid: Editorial de la Universidad Complutense.

KLAASEN, L.M. (1974): "Prelude opeen recreative Kostenbaten analyse", Recr. Voorz, 3.

McEVOY, J. (1974): "Hours of work ant the demand for outdoor recreation", Journal of Leisure Research, 6.

MIOSSEC, J.M. (1976): "Eléments pour une théorie de l'espace touristique", Les Calliers du Tourisne, serie C, 36 .

REILLY, W.J. (1929): "Methods for the study of retail relationships", University of Texas Bulletin, 2944.

SMITH, S.L.J. (1983): "Identification of functional tourism regions in North America", Journal of Travel Research, 22(4), 13-21.

STEWART, J.Q. (1950): "The development of social physics", American Journal of Physics, 18, 239253.

VAN DOREN, C.S. (1967): An Interaction Travel Model for Projecting Attendance of Campers at Michigan State Parks: a Study in Recreational Geograplly. Michigan: State University. 
VAN LIER, H.N. (1977): "Improvement of Demand Studies as Tool for Planning Outdoor Recreation", State of the Art Methods for Research. Planning and Determining the benefits of Outdoor Recreation. Berkeley: Pacific Southwest Forest and Range Experiment Station.

WOLFE, R.I. (1972): "The inertia model", Journal of Leisure Research, 4.

ZIPF, G.K. (1949): Hunan behavior and the principle of least effort. Cambridge: Addison-Wesley Press. 